\title{
POINTWISE COMPACTNESS ON EXTREME POINTS
}

\author{
SURJIT SINGH KHURANA
}

\begin{abstract}
It is proved that if $X$ is a compact convex subset of a locally convex space $E$, then the subset $S$ of $A$, the space of all affine, scalar-valued continuous functions on $X$, which is uniformly bounded on $X$ and relatively countably compact in the pointwise topology on $\operatorname{ext}(X)$, is relatively compact in the pointwise topology on $X$.
\end{abstract}

In [1] it is proved that a bounded subset of a Banach space is relatively weakly compact if it is relatively countably compact relative to the topology of pointwise convergence on the extreme points of the closed unit ball of the dual space. In this note we prove that it is a simple consequence of the Choquet integral representation theorem [2] and the fact that the relatively pointwise countably compact sets of continuous functions on $\sigma$-compact spaces are angelic spaces [3].

All locally convex spaces are on $K$, the field of real or complex numbers. For any topological space $Y, C(Y)$ is the space of all continuous $K$-valued functions on $Y$. By a $\sigma$-compact set we mean a countable union of compact sets.

THEOREM 1. Let $X$ be a compact convex subset of a Hausdorff locally convex space $E, A$ the vector space of all affine, continuous, $K$-valued functions on $X$, and $S a$ subset of $A$, uniformly bounded on $X$. If $S$ is a relatively countably compact subset of $A$, in the topology of pointwise convergence on $\operatorname{ext}(X)$, the extreme points of $X$, then $S$ is relatively compact in the topology of pointwise convergence on $X$.

Proof. Without loss of generality, we assume that $X$ is contained in a closed hyperplane of $E$, which misses the origin and $E=$ space generated by $X[5$, p. 59]. By defining the value at the origin equal to 0 , every element of $A$ can be uniquely extended so as to become an element of $E^{\prime}$, the topological dual of $\left(E, \sigma\left(E, E^{\prime}\right)\right)$ (note $X$ is still compact); thus we identify $A$ with $E^{\prime}$.

Let $\tau_{1}$ and $\tau_{2}$ be the topologies, on $E^{\prime}$, of pointwise convergence on $X$ and ext $X$. Fix a sequence $\left\{f_{n}\right\} \subset S$ and $f_{\infty} \in E^{\prime}$ be a $\tau_{2}$-cluster point of $\left\{f_{n}\right\}$. We shall prove that it is also a $\tau_{1}$-cluster point. This will prove the result ([3]; note $\left.S \subset C(X)\right)$. Suppose $f_{\infty}$ is not a $\tau_{1}$-cluster point of $\left\{f_{n}\right\}$. Thus there exist a subnet $\left\{f_{\alpha}\right\} \subset\left\{f_{n}\right\}$, an $x \in X$, and an $\varepsilon>0$ such that $f_{\alpha} \rightarrow f_{\infty}$ in $\tau_{2}$-topology, but

$$
f_{\alpha}(x)>f_{\infty}(x)+\varepsilon, \text { for every } \alpha
$$

or $f_{\alpha}(x)<f_{\infty}(x)-\varepsilon$, for every $\alpha$.

Received by the editors October 5, 1980 and, in revised form, November 20, 1980.

1980 Mathematics Subject Classification. Primary 46A50, 46A55; Secondary 52A07.

Key words and phrases. Convex sets, barycenters, extreme points, integral representation.

() 1981 American Mathematical Society 0002-9939/81/0000-0476/\$01.50 
Taking $F=\cap_{1<n<\infty} f_{n}^{-1}\{0\}$, we get a canonical mapping $\varphi: E \rightarrow E_{1}=E / F$, with quotient topology; $X_{1}=\varphi(X)$ is a metrizable, compact, convex subset of $E_{1}$ and $\left\{f_{n}: 1 \leqslant n \leqslant \infty\right\} \subset E_{1}^{\prime}$. Take a probability measure $\mu$ on $X_{1}$ such that $\mu\left(\right.$ ext $\left.X_{1}\right)=1$ and the barycenter of $\mu$ is $\varphi(x)$ [2]. Thus there exists a $\sigma$-compact set $C_{1} \subset$ ext $X_{1}$ such that $\mu\left(C_{1}\right)=1$. Considering $\varphi: X \rightarrow \varphi(X), C=\varphi^{-1}\left(C_{1}\right)$ is a $\sigma$-compact subset of $X$. For $y \in C_{1} \subset$ ext $X_{1}, \varphi^{-1}(y)$ is an extremal [4] subset of $X$ and so $\operatorname{ext}\left(\varphi^{-1}(y)\right) \subset \operatorname{ext} X$. This means that if $g \in E^{\prime}$ is a $\tau_{2}$-limit of a net $\left\{f_{\beta}\right\} \subset\left\{f_{n}\right\}$, in $S$, then by Krein-Milman theorem and the continuity of $g, f_{\beta} \rightarrow g$, pointwise on $C$. Thus $\left\{f_{n}\right\}_{\mid C}$ is relatively countably compact with the topology of pointwise convergence on $C$. By [3, Theorem 2.1], there exists a subsequence $\left\{f_{n(k)}\right\} \subset\left\{f_{\alpha}\right\}$, with $f_{n(k)} \rightarrow f_{\infty}$ pointwise on $C$. By the dominated convergence theorem $\mu\left(f_{n(k)}\right) \rightarrow \mu\left(f_{\infty}\right)$ and so $f_{n(k)}(x) \rightarrow f_{\infty}(x)$, a contradiction. The theorem is proved.

Corollary 2 [1, Theorem 1]. Let $G$ be a Banach space, $G^{\prime}$ its dual, $B$ the closed unit ball of $G^{\prime}$, and $Y$ a bounded, relatively countably compact, in the topology of pointwise convergence on $\operatorname{ext}(B)$, subset of $G$. Then $G$ is relatively weakly compact.

Proof. Take $E=\left(G^{\prime}, \alpha\left(G^{\prime}, G\right)\right), X=B$ and $S=Y$. The result follows from Theorem 1.

\section{REFERENCES}

1. J. Bourgain and M. Talagrand, Compacité extrêmale, Proc. Amer. Math. Soc. 80 (1980), 68-70.

2. G. Choquet, Lectures on analysis, Benjamin, New York, 1969.

3. J. D. Pryce, A device of $R$. J. Whitley's applied to pointwise compactness in function spaces, Proc. London Math. Soc. (3) 23 (1971), 532-546.

4. J. D. Pryce and D. H. Fremlin, Semi-extremal sets and measure representation, Proc. London Math. Soc. (3) 29 (1974), 502-520.

5. R. R. Phelps, Lectures on Choquet theorem, Van Nostrand, Princeton, N. J., 1966.

Department of Mathematics, University of Iowa, Iowa CtTy, Iowa 52242 